\title{
Dynamique de la régénération naturelle des essences commerciales sur les routes secondaires de différents âges de l'UFA 09-003 (Djoum, Cameroun)
}

\author{
E. M. AMBOMBO ONGUENE ${ }^{1}$, J. B. NGODO MELINGUI ${ }^{1}$, A. C. PIAL ${ }^{2 *}$, \\ A. MBARGA BINDZI ${ }^{1}$ et D. C. MOSSEBO ${ }^{3}$
}

\author{
${ }^{I}$ Université de Yaoundé I, Faculté des Sciences, Département de Biologie et Physiologie Végétales, \\ Laboratoire de Botanique-Ecologie ; B.P : 812 Yaoundé, Cameroun. \\ ${ }^{2}$ Université de Yaoundé I, Faculté des Sciences, Département de Biologie et Physiologie Végétales, \\ Laboratoire des Biotechnologies Végétales et Environnement ; B.P : 812 Yaoundé, Cameroun. \\ ${ }^{3}$ Université de Yaoundé I, Faculté des Sciences, Département de Biologie et Physiologie Végétales, \\ Laboratoire de Cryptogames ; B.P : 812 Yaoundé, Cameroun. \\ *Auteur correspondants ; E-mail : annieclaude_pial@hotmail.com
}

\section{RESUME}

Le Cameroun dispose d'une superficie de forêt estimée à 22 millions d'hectares qui assure des fonctions économiques, sociales et culturelles. Compte tenu de l'importance des services rendus, la forêt camerounaise devrait être gérée de manière durable, ce qui n'est pas encore le cas. L'étude menée dans l'Unité Forestière d'Aménagement (UFA) 09-003 à Djoum avait pour objectif d'évaluer la régénération naturelle des essences commerciales sur les routes secondaires de différents âges. L'échantillonnage d'une superficie de 0,9 ha s'est fait à l'aide de 90 placettes de $100 \mathrm{~m}^{2}(10 \mathrm{~m}$ x $10 \mathrm{~m})$, le long des 20 premiers mètres des routes secondaires d'1 an ; 5 ans et 15 ans d'âge. Les paramètres tels que la dominance, l'importance relative, la densité relative, les indices de Simpson et de Shannon, la chorologie des diaspores ont été étudiés. Les résultats d'inventaire montrent un enrichissement progressif en essences héliophiles (Terminalia superba, Nauclea diderrichii, Distemonanthus benthamianus et Alstonia boonei) sur les forêts semi-ouvertes à Marantaceae et Zingiberaceae de 1 à 15 ans d'âge et en essences semi-sciaphiles (Desbordesia glaucescens) et sciaphiles (Staudtia kamerunensis) qui font leur apparition 15 ans après l'exploitation forestière. Ces différentes espèces présentent une régénération faible dans l'ensemble et l'anémochorie est le mode principal de dispersion des diaspores, suivie de la zoochorie. Pour favoriser la régénération naturelle des espèces sur les routes secondaires au moins un an après l'exploitation, les sociétés forestières devraient éviter l'envahissement de ces sites par les graminées, favoriser la croissance des espèces héliophiles par des défrichements réguliers des bordures, opérer un monitoring systématique des essences et sensibiliser les populations riveraines sur la nécessité de maintenir la dynamique de régénération de ces espèces.

(c) 2018 International Formulae Group. All rights reserved.

Mots clés : Exploitation forestière, essences commerciales, routes secondaires, dynamique de la régénération, Unité Forestière d'Aménagement.

\section{Dynamics of the natural regeneration of commercial species on the secondary roads of different ages in 09-003 FMU (Djoum, Cameroon)}




\section{ABSTRACT}

Cameroon has a forest area production estimated at 22 million ha which provides economic, social and cultural functions. Given the importance of the services handed, the Cameroonian forest should be managed in a sustainable way. It is not actually the case regarding the state of degradation of the Cameroonian forest. The study carried out in the Djoum Forest Management Unit (FMU) 09-003 aimed at evaluating the natural regeneration of commercial species on secondary roads of different ages. Sampling of 0.9 ha was carried out using 90 plots of $100 \mathrm{~m}^{2}(10 \mathrm{~m} \times 10 \mathrm{~m})$ along the first $20 \mathrm{~m}$ of the secondary roads of 1,5 and 15 years of age. Parameters such as dominance, relative importance, relative density, Simpson and Shannon indices, diaspora chorology were studied. The inventory shows a gradual enrichment in heliophilous species (Terminalia superba, Nauclea diderrichii, Distemonanthus benthamianus and Alstonia boonei) on semi-open forests of Marantaceae and Zingiberaceae from 1 to 15 years, semi-sciaphil (Desbordesia glaucescens) and sciaphil species (Staudtia kamerunensis) which appear 15 years after logging. These different species showed an overall weak regeneration potentiality while anemochoria is the main way of diaspora dispersal, followed by zoochoria. To encourage natural regeneration of commercial species on secondary roads at least one year after harvesting, forestry companies should regularly destroy invasing grasses, encourage the growth of heliophilic species through regular clearing of roads borders, conduct systematic monitoring of species and sensitize local populations on the need to maintain the regeneration dynamics of these species.

(C) 2018 International Formulae Group. All rights reserved.

Keywords: Logging, commercial species, secondary roads, dynamics of regeneration, Forest Management Unit.

\section{INTRODUCTION}

Le Cameroun dispose d'une étendue de forêts de production évaluée à 22 millions d'ha dont 17,5 millions sont exploitables (MINFOF, 2016). Au niveau national, cette forêt assure des fonctions économique, sociale et culturelle et représente, sur le plan économique, $4 \%$ du PIB. Par ailleurs, son rôle dans la lutte contre les changements climatiques à travers la séquestration du carbone est établi par de nombreuses recherches (Bele et al., 2011 ; Pandey et al., 2016).

Au regard de l'importance des services rendus, la forêt camerounaise mérite d'être gérée de manière durable. Ce n'est malheureusement pas encore le cas, car on observe qu'elle se dégrade au fil du temps et il est prévu que le Cameroun perde plus de 2,018 millions d'ha de forêts à l'horizon 2035 (Tchatchou et al., 2015). L'exploitation forestière à elle seule entraîne la fragmentation et la réduction du massif forestier, conséquence de l'ouverture des voies d'accès (développement d'infrastructures routières), de la création des parcs à bois, de l'installation de chantiers et de l'afflux des populations (Ndobe et Mantzel, 2014). Cette exploitation entraîne également la réduction et l' appauvrissement en espèces de bois d'œuvre (Ngueguim et al., 2015). Bien qu'elle concerne principalement les espèces commerciales, l'exploitation forestière peut, en fonction de son intensité et des techniques utilisées, avoir des conséquences diverses sur l'ensemble de l'écosystème notamment sur la régénération des espèces (Missamba-Lola et al., 2015).

Dans la perspective de gérer durablement les espèces visées par l'exploitation forestière, une étude a été menée dans l'UFA 09003 pour évaluer leur facilité de régénération sur les routes secondaires de différents âges, soit 1,5 et 15 ans.

\section{MATERIEL ET METHODES Présentation du site d'étude}

L'étude a été menée dans l'UFA 09 003 de la forêt de production de la SFID située dans l'arrondissement de Djoum ( $2^{\circ} 39^{\prime} 50^{\prime \prime}$ de latitude Nord et les méridiens $12^{\circ} 40^{\prime} 02^{\prime \prime}$ de longitude Est), département du Dja et Lobo, région du Sud Cameroun. Cette 
UFA possède un réseau routier dense, constitué d'axes de liaison inter-sites, de routes principales et secondaires d'exploitation (Figure 1). Ces dernières sont utilisées sur une courte période pour faciliter l'extraction $\mathrm{du}$ bois dans les zones d'exploitation.

\section{Collecte des données \\ Choix des routes secondaires}

Les routes secondaires sont, après les pistes de débusquage et de débardage, des éléments essentiels de l'exploitation forestière. Celles de cette étude ont été repérées grâce aux anciennes cartes d'aménagement. Le critère «accessibilité »a guidé le choix des routes secondaires qui ont été classées en fonction de leurs âges d'ouverture.

\section{Préparation des placettes}

Les placettes de forme carrée, $100 \mathrm{~m}^{2}$ $(10 \mathrm{~m} \times 10 \mathrm{~m})$ ont été constituées aux abords immédiats des routes secondaires, pour limiter l'influence des peuplements adjacents (Doucet, 2003). Il subsiste en général, au-delà de $20 \mathrm{~m}$ de la route, après ouverture, un grand nombre d'arbres et d'arbustes du peuplement antérieur qui pourrait modifier le résultat au cas où ils sont inclus (Missamba-Lola et al., 2015).

Les placettes ont été disposées selon un plan aléatoire temporel de l'ouverture des routes secondaires : les trois périodes retenues correspondaient aux années 2013-2014 (2-1 ans) ; 2010-2011 (5-4 ans) ; 2000-2001 (15-14 ans) à raison de 30 relevés par période dont 15 relevés par année d'exploitation. Ainsi, 90 placettes ont été installées au hasard le long des routes secondaires selon le dispositif de la Figure 2.

\section{Inventaire}

La méthode d'inventaire systématique de toutes les essences commerciales $(h>10$ $\mathrm{cm})$ rencontrées sur les différentes placettes a été effectuée.

L'équipe de comptage était composée :

- d'un pointeur qui collectait les données à l'aide d'une fiche de collecte par placette ;
- de deux compteurs qui identifiaient les espèces selon une nomenclature vernaculaire (Bulu, Ewondo) et commerciale, mesuraient la hauteur des plantules, arbustes et arbres à l'aide d'une perche de $2 \mathrm{~m}$ graduée en dizaine de $\mathrm{cm}$, et le diamètre des tiges rencontrées à l'aide d'un pied à coulisse.

Lorsque l'identification d'une espèce était impossible sur site, un échantillon était prélevé, ramené au laboratoire pour identification selon Vivien et al. (1985).

Toutes les tiges des essences commerciales de hauteur $\geq 2 \mathrm{~m}$ ont été inventoriées sur $40 \mathrm{~m}^{2}(10 \mathrm{~m} \mathrm{x} 4 \mathrm{~m})$ de part et d'autre de la bande centrale de $20 \mathrm{~m}^{2}$, la mesure de la hauteur et du diamètre à hauteur de poitrine $(\mathrm{Dhp}=1,30 \mathrm{~m})$ a été relevée. Sur la bande centrale de $20 \mathrm{~m}^{2}(2 \mathrm{~m} \mathrm{x} 10 \mathrm{~m})$, l'inventaire des essences commerciales de hauteur comprises entre $10 \mathrm{~cm}$ et $2 \mathrm{~m}$ a été réalisé.

\section{Analyse des données}

Les données collectées ont été saisies, apurées et analysées à l'aide du progiciel $\mathrm{R}$ version 3.1.3. Les graphiques ont été réalisés grâce au logiciel Excel office professionnel 2010.

L'importance d'une espèce a été estimée en fonction de l'abondance ou du nombre d'individus de l'espèce; de la dominance ou de la surface terrière de l'espèce; de la fréquence ou du nombre de placettes dans lesquelles l'espèce est présente.

L'indice d'importance relative (Ir) a été déterminé selon Doucet (2003). Il correspond à la somme de sa densité, de sa dominance et de sa fréquence relative. Cet indice est compris entre 0 et 300 .

La composition du peuplement en terme de famille a été déterminée grâce à l'indice de diversité relative (Doucet, 2003).

Diversité relative $=$

$$
100 \times \frac{\text { Nombre d'espèces au sein } \mathrm{d}^{\prime} \text { une famille }}{\text { Nombre total d'espèces échantillonnées }}
$$

Les indices de Simpson (Dsi) et de Shannon $(\mathrm{H})$ ont permis les mesures de diversité dans chacune des périodes retenues. L'indice de Simpson est interprété comme la 
probabilité que deux individus tirés au hasard soient d'espèces différentes (Marcon, 2016). Lorsque la diversité est maximale, sa valeur est de 0 , lorsque la diversité est minimale, elle est de 1 (Doucet, 2003).

$\mathrm{H}=-\sum_{i=1}^{S} P i . \log 2(P i)$

L'indice de Shannon mesure la diversité spécifique d'un peuplement, en combinant l'abondance relative des espèces et la richesse spécifique (Tiebre et al., 2016). Cet indice varie de 0 (une seule espèce) à $\ln$ $\mathrm{S}$ (toutes les espèces ont la même abondance), S étant le nombre d'espèces, Doucet (2003).
$\mathrm{D}_{\mathrm{Si}}=1-\sum_{i=1}^{S} P^{2} i$

$i$ : une espèce du milieu étudié

$\mathrm{p}_{i}$ : proportion d'une espèce $i$ par rapport au nombre total d'espèces $(\mathrm{S})$ dans le milieu étudié dont le calcul se fait selon la formule : $\mathrm{p}(i)=\mathrm{n}_{i} / \mathrm{N}$ avec $\mathrm{n}_{i}:$ nombre d'individus pour l'espèce $i$ et $\mathrm{N}$ est l'effectif total. Par ailleurs, les densités spécifiques de chaque période ont permis, sur la base des âges des routes secondaires, d'identifier les essences commerciales en régénération acquise ( $d$ 'au moins $2 \mathrm{~m}$ de hauteur) et en régénération installée (inférieure à $2 \mathrm{~m}$ de hauteur).

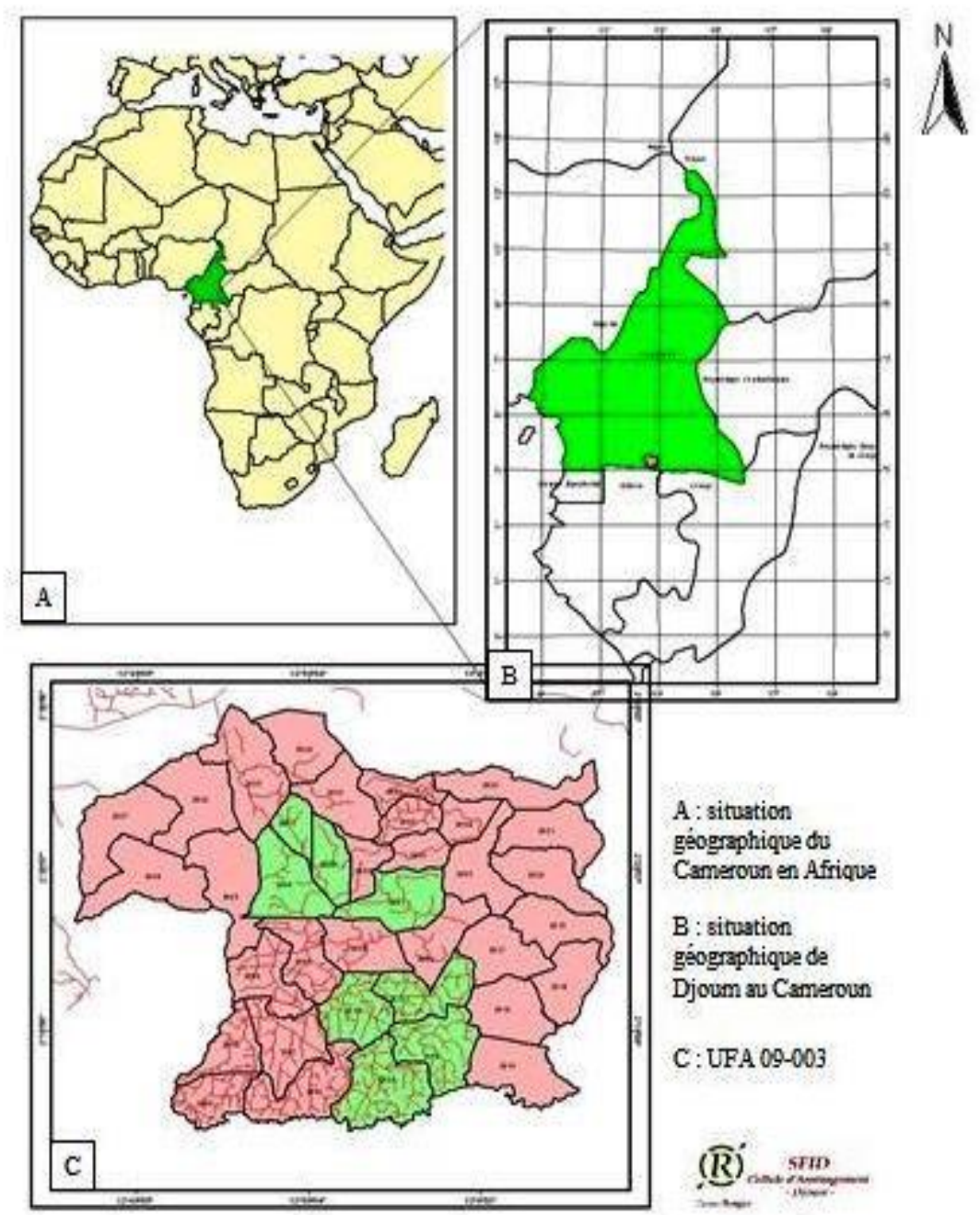

Figure 1 : Carte de l'UFA 09003 (SFID-Djoum, 2015). 


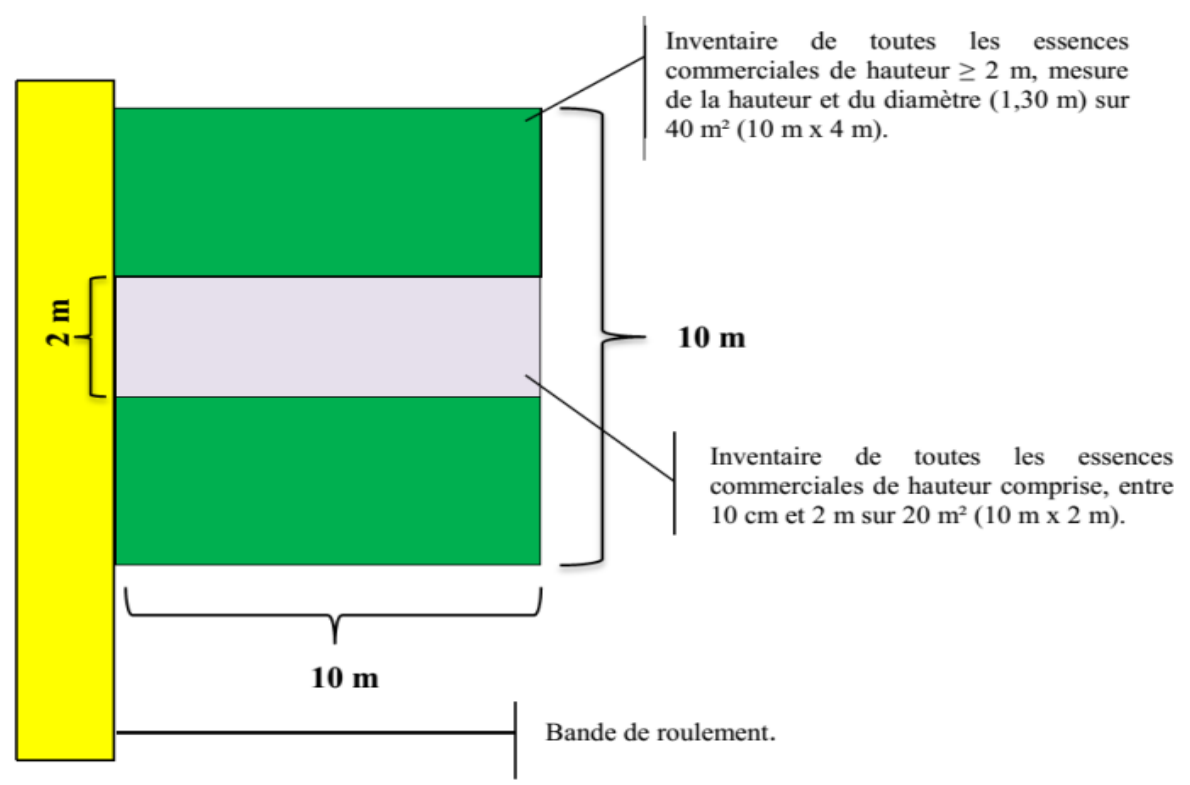

Figure 2 : Dispositif expérimental d'inventaire (Missamba-Lola et al., 2015).

\section{RESULTATS}

\section{Diversité floristique des essences commerciales sur les routes secondaires}

En terme de diversité floristique, dixhuit familles ont été dénombrées dans les 90 placettes $(\mathrm{h}>2 \mathrm{~m})$, six parmi elles sont les plus importantes, comme le démontre la Figure 3.

En terme d'abondance, on a les Irvingiaceae représentées par Desbordesia glaucescens (23 individus) et Klainedoxa sp (01 individus), puis les Combretaceae qui possèdent des valeurs élevées en densité malgré la dominance d'une seule espèce Terminalia superba (17 individus).

L'analyse des paramètres de richesse et de diversité floristique a permis de mettre en relief les variations du peuplement selon les différentes périodes étudiées. La richesse spécifique est plus élevée 5 ans après l'exploitation forestière (indice de Simpson le plus bas) comparée à 1 et 15 ans. La forêt de 15 ans (en cours de fermeture) présente une diversité floristique importante (indice de Shannon élevé) comparativement à la forêt semi-ouverte d' 1 an et 5 ans.

\section{Composition floristique des essences commerciales par période}

Sur les placettes de 1 à 5 ans, en forêt semi-ouverte à Marantaceae et Zingiberaceae, on note l'émergence des Euphorbiaceae arborescentes pionnières avec prédominance de Terminalia superba (Ir = 0,55), seule essence commerciale suffisamment bien représentée. Par endroits, on observe des parasoliers (Musanga cecropioides) ainsi que des lianes à eau (Tetracera alnifolia). Les autres espèces de la famille des Euphorbiaceae tendent à disparaître.

Après 15 ans, les espèces spécifiques de forêt sempervirente sont largement dominantes et sont représentées par Desbordesia glaucescens $(\mathrm{Ir}=24,18)$, Fagara sp $(\operatorname{Ir}=12,32)$; ainsi la famille des Irvingiaceae est largement dominante. On assiste aussi à l'apparition des espèces ligneuses de sous-bois à tendance sciaphile : Staudtia kamerunensis, quelques individus de parasoliers (Musanga cecropioides) persistent.

Les Figures 4 et 5 présentent la densité des essences à fortes valeurs commerciales rencontrées dans les différentes placettes. La Figure 4 présente les individus de hauteur 
supérieure à $2 \mathrm{~m}$ tandis que la Figure 5 présente la régénération naturelle installée (h $<2 \mathrm{~m}$ ) sur les routes secondaires.

La présence des essences exploitées est observée, à des proportions diverses. Distemonanthus benthamianus, Desbordesia glaucescens (à partir de 15 ans), Bombax buonopozense et Bombax brevicuspe (1 tige/ha) ont dans l'ensemble une faible régénération. Les espèces qui s'installent dès l'ouverture de la route sont Nauclea diderrichii (48 tiges/ha), Alstonia boonei et Erythrophleum ivorense. Staudtia kamerunensis et Desbordesia glaucescens font leur apparition 15 ans après l'exploitation dans le sous-bois des zones de forêts fermées.

Bien que la modification de la composition floristique le long des routes secondaires de 1 à 15 ans soit effective, on remarque un enrichissement progressif en essences héliophiles. Desbordesia glaucescens pousse presque exclusivement sur les routes secondaires de forêt semi-ouverte de 15 ans tandis que Terminalia superba semble tolérer les routes secondaires de tout âge. L'émergence des sciaphiles s'observe seulement 15 ans après l'ouverture de la route secondaire quand le milieu se referme déjà tendant à faire entrer la lumière par faisceaux.

\section{Dynamique de la végétation sur les routes secondaires après exploitation}

Quatre cent quinze tiges d'espèces commerciales, correspondant à 22, espèces ont été dénombrées dont 308 tiges d'individus avec une hauteur inférieure à $2 \mathrm{~m}$ et 107 tiges d'individus dont la hauteur dépasse $2 \mathrm{~m}$ (Figure 6). Les individus de faibles diamètres sont dominants et représentés par les herbacées issues des familles Marantaceae et les Zingiberaceae.

Le nombre moyen des tiges $(\mathrm{h}>2 \mathrm{~m})$ sur la totalité des placettes est de 1,2 tige par placette avec un nombre moyen plus important dans les placettes de 15 ans d'âge (3,4 tiges) et presque nul pour les placettes de 1 et 5 ans d'âge (Figure 7).

\section{Mode de dispersion des essences commerciales sur les routes secondaires}

Les principaux modes de dissémination sont : zoochore (endozoochore et épizoochore), autochore et anémochore (Figure 8). Les placettes dont l'ouverture est récente (1 à 5 ans) sont plus concernées par l'anémochorie et la zoochorie du fait que les routes récemment ouvertes sont envahies par les déjections d'animaux. Celles de 15 ans d'âge sont dominées par l'anémochorie, la dispersion de Desbordesia glaucescens et Petersianthus macrocarpus. Quant à l'autochorie, elle se rencontre à tous les différents âges.

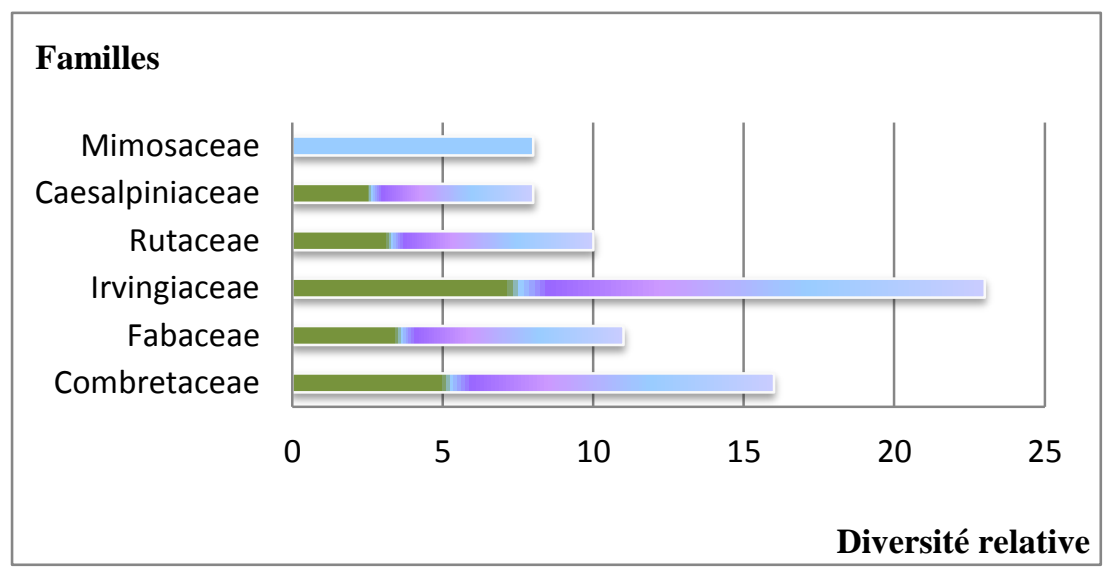

Figure 3 : Familles les plus importantes dans les 90 placettes. 


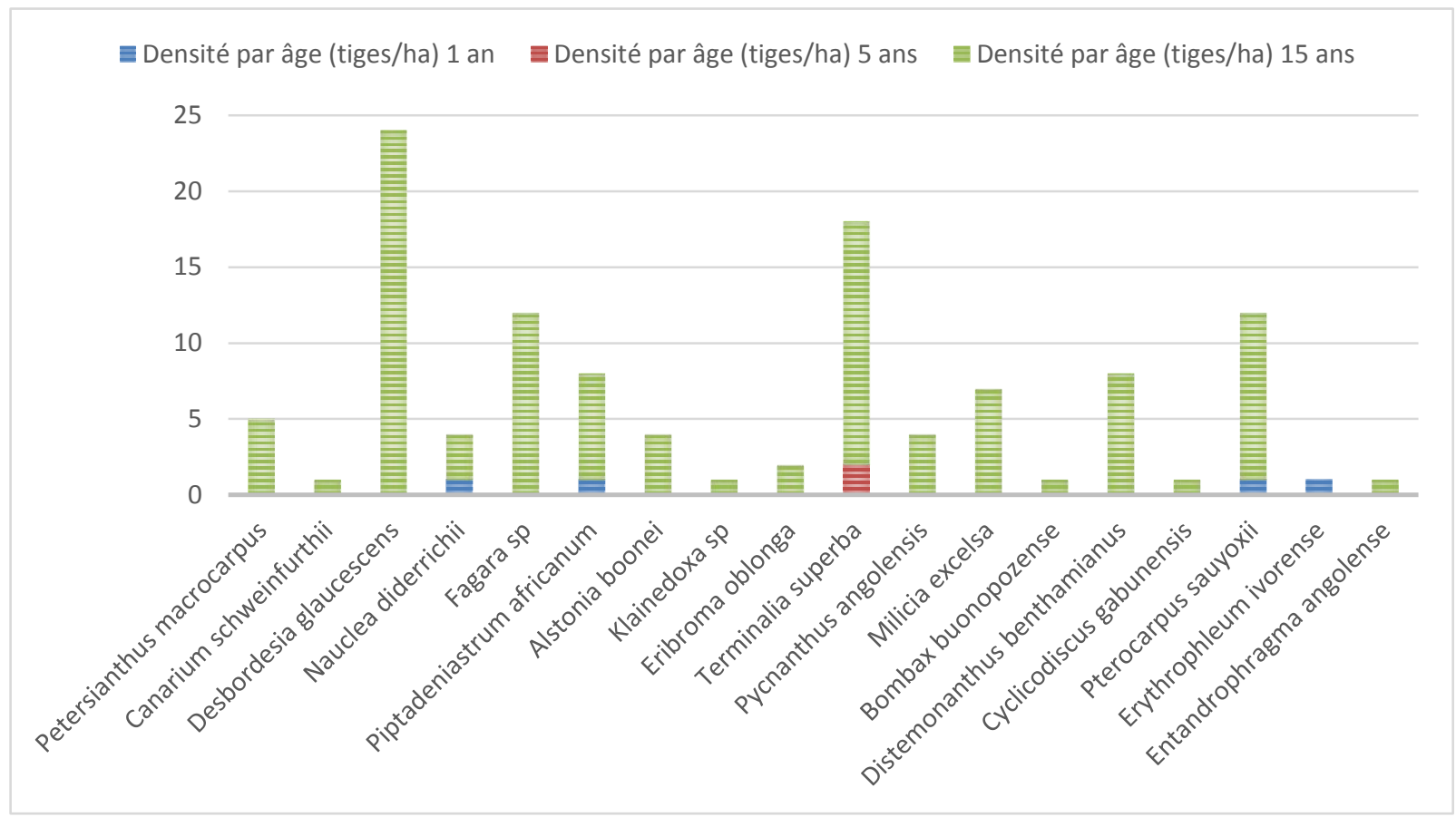

Figure 4 : Evolution de la densité de régénération naturelle acquise $(\mathrm{h}>2 \mathrm{~m})$.

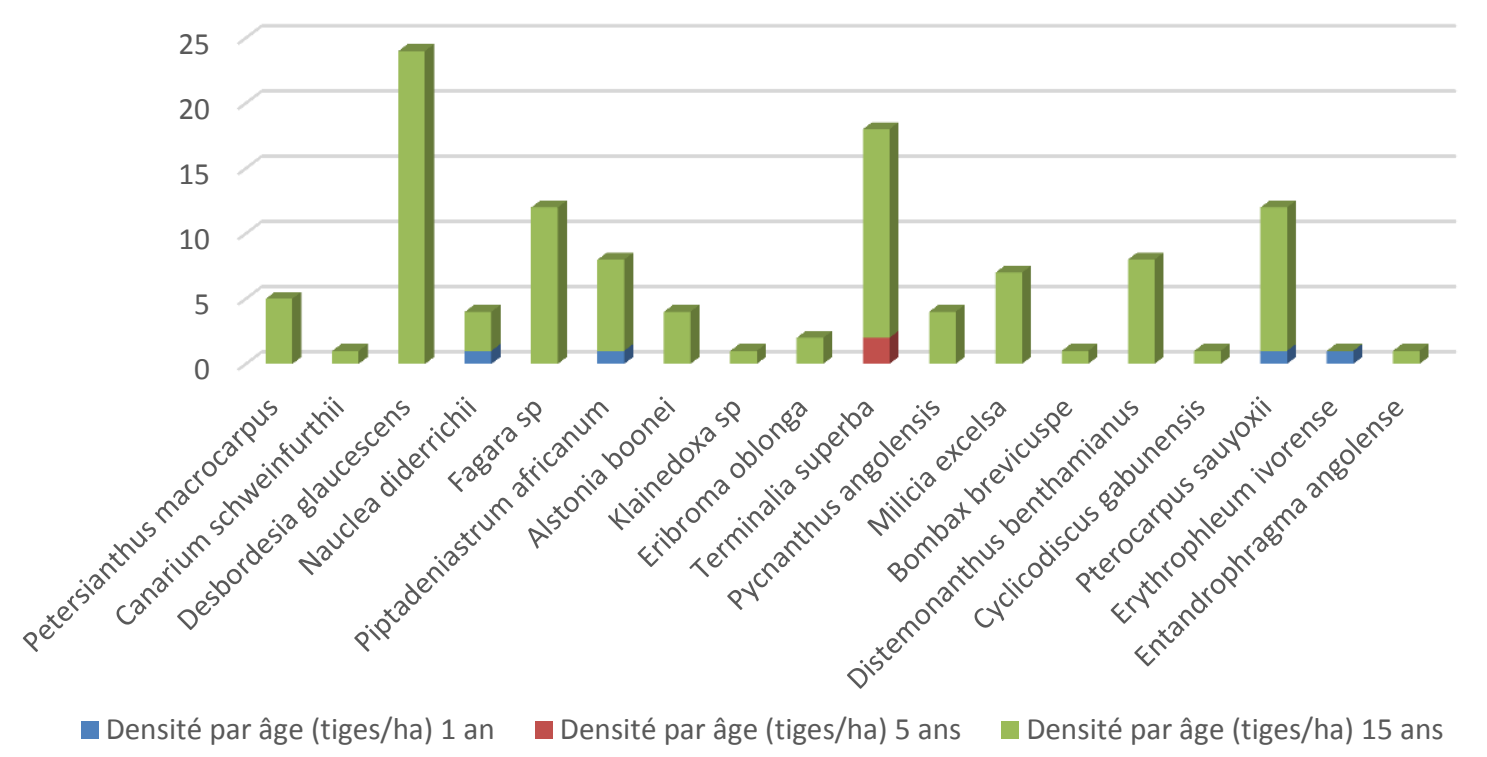

Figure 5 : Evolution de la densité de régénération naturelle installée ( $h<2 \mathrm{~m})$. 


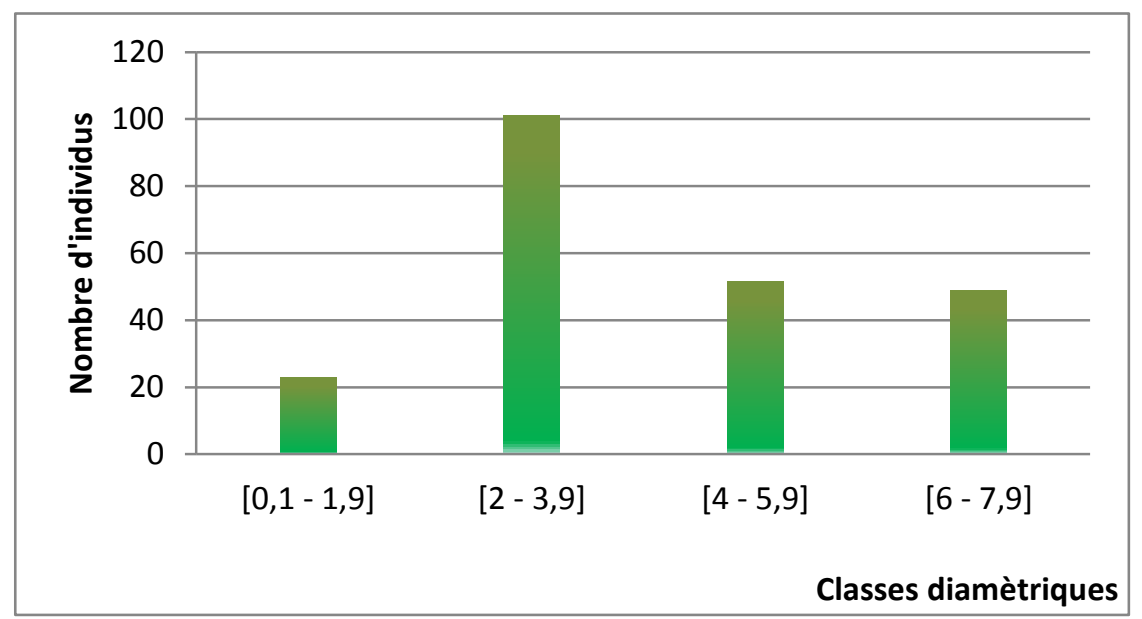

Figure 6 : Nombre d'individus en fonction des classes de diamètres dans les placettes ( $\mathrm{h}>2 \mathrm{~m})$.

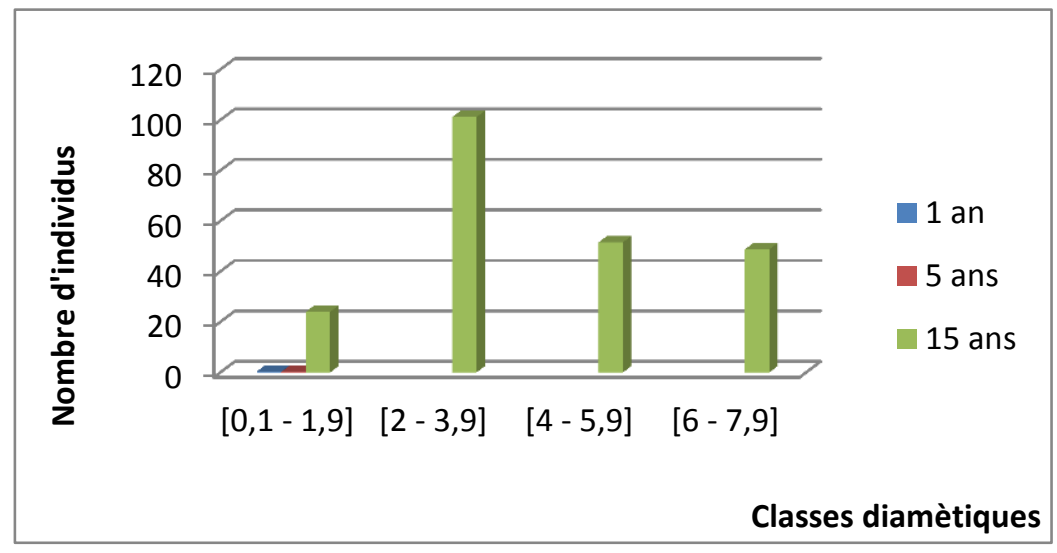

Figure 7 : Nombre d'individus en fonction des classes diamètriques par âge dans les placettes.

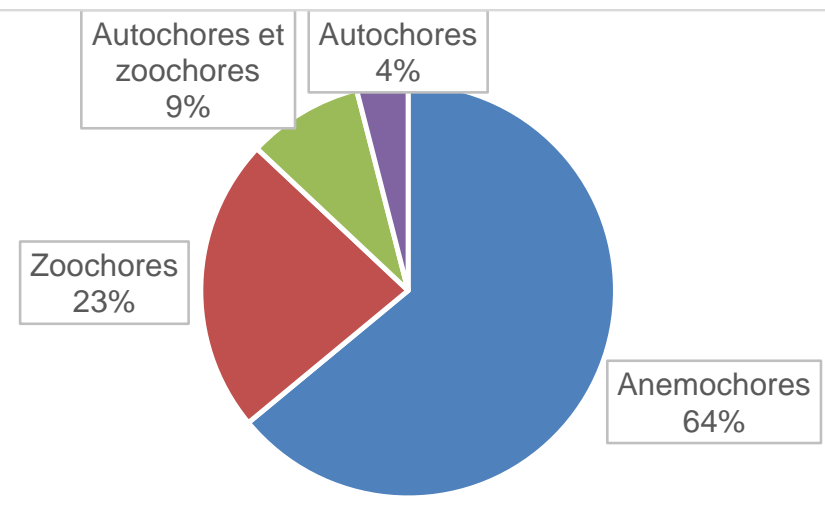

- Anemochores - Zoochores - Autochores et zoochores - Autochores

Figure 8 : Distribution des types chorologiques sur les routes secondaires. 
Tableau 1 : Indices calculés par période d'âge.

\begin{tabular}{lccc}
\hline Périodes & $\mathbf{1}$ à $\mathbf{2}$ ans & $\mathbf{4}$ à $\mathbf{5}$ ans & $\mathbf{1 4}$ à $\mathbf{1 5}$ ans \\
\hline Paramètres & & & \\
Nombre de tiges & 146 & 75 & 194 \\
Indice de Simpson & 0,79 & 0,62 & 0,86 \\
Indice de Shannon & 1,80 & 1,16 & 2,38 \\
\hline
\end{tabular}

Tableau 2 : Importance relative des espèces par période post-exploitation.

\begin{tabular}{|c|c|c|c|c|}
\hline \multirow[t]{2}{*}{$\mathbf{N}^{\circ}$} & \multirow[t]{2}{*}{ Espèce } & \multicolumn{3}{|c|}{ Importance relative } \\
\hline & & 1 à 2 ans & 4 à 5 ans & 14 à 15 ans \\
\hline 1 & Alstonia boonei & 0,35 & 0,06 & 0,53 \\
\hline 2 & Bombax brevicuspe & 0 & 0 & 0,09 \\
\hline 3 & Bombax buonopozense & 0 & 0 & 0,04 \\
\hline 4 & Canarium schweinfurthii & 0 & 0 & 0,17 \\
\hline 5 & Cyclicodiscus gabunensis & 0 & 0,07 & 0,10 \\
\hline 6 & Desbordesia glaucescens & 0 & 0 & 24,18 \\
\hline 7 & Distemonanthus benthamianus & 0,04 & 0,51 & 2,46 \\
\hline 8 & Entandrophragma angolensis & 0 & 0 & 0,05 \\
\hline 9 & Eribroma oblonga & 0,12 & 0 & 0,35 \\
\hline 10 & Erythrophleum ivorense & 0,22 & 0 & 0,08 \\
\hline 11 & Fagara heitzii & 0 & 0 & 0,04 \\
\hline 12 & Fagara sp & 0 & 0 & 12,32 \\
\hline 13 & Klainedoxa sp & 0,04 & 0 & 0,06 \\
\hline 14 & Lovoa trichilioides & 0,04 & 0 & 0 \\
\hline 15 & Milicia excelsa & 0,12 & 0 & 2,26 \\
\hline 16 & Nauclea diderrichii & 0,39 & 0,05 & 0,89 \\
\hline 17 & Petersianthus macrocarpus & 0 & 0,12 & 1,19 \\
\hline 18 & Piptadeniastrum africanum & 0,06 & 0 & 3,86 \\
\hline 19 & Pterocarpus soyauxii & 0,05 & 0 & 3,46 \\
\hline 20 & Pycnanthus angolensis & 0 & 0 & 0,64 \\
\hline 21 & Staudtia kamerunensis & 0 & 0 & 0,18 \\
\hline 22 & Terminalia superba & 0,15 & 0,55 & 19,84 \\
\hline
\end{tabular}




\section{DISCUSSION}

La gestion durable des espèces forestières suppose que leur facilité de régénération soit maitrisée, particulièrement au niveau des routes secondaires, les trouées d'abattage ou les parcs, zones de régénération potentielle de la banque des graines du sol. L'étude menée dans l'UFA 09003 de la SFID avait pour objectif d'évaluer la capacité de régénération naturelle des essences commerciales sur les routes secondaires de différents âges, 1, 5 et 15 ans.

La diversité floristique est faible dans l'ensemble du site d'étude. Ce résultat serait la conséquence de la dominance des Marantaceae et des Zingiberaceae après l'ouverture du milieu. Il concorde avec ceux de Doucet (2003) qui conclut dans son étude que les ouvertures du couvert forestier, comme les routes secondaires favorisent le développement des Marantaceae et Zingiberaceae, lesquelles forment un couvert difficilement pénétrable inhibant ou ralentissant fortement la régénération des espèces ligneuses. D'autres auteurs (FAO, 2010 et Yongo et al., 2013) associent à cela, l'érosion des sols qui en diminue la fertilité et peut gêner la régénération naturelle de la végétation et donc aggraver la perte de biodiversité.

Selon l'évolution structurale et biologique d'une parcelle abandonnée, les premières plantes qui occupent l'espace sont les herbacées graminées pour la plupart, ensuite s'installe l'espèce Chromolaena odorata susceptible d'être associée à d'autres herbacées pérennes telles que: Panicum maximum, Phyllanthus amarus et autres. L'étude de la dynamique des populations est essentielle pour le développement de scénarii de gestion rationnelle des écosystèmes forestiers (Akouehou, 2017). Ainsi, la dynamique de la végétation présente une faible densité en terme de nombre d'individus de faible diamètre correspondant à une surface terrière de $8,6 \mathrm{~m}^{2} / \mathrm{ha}, 415$ individus ont été dénombrés. Des résultats similaires ont été obtenus par Missamba-Lola et al. (2015) dans une étude réalisée au Congo et Doucet (2003) montrant que sur les routes secondaires des forêts anthropisées, les individus sont majoritairement de faibles diamètres. En effet, la réduction du nombre d'individus du stade plantule vers le stade grand arbre s'explique par l'influence des facteurs écologiques et anthropiques qui sont à l'origine des mortalités massives observées dans les différentes populations végétales (Koubouana et al., 2016). On observe une régénération faible de l'ensemble des essences commerciales rencontrées, situation qui peut être inversée par la mise en œuvre de techniques sylvicoles appropriées qui contribueront à leur maintien dès leur germination.

Quatre familles sont les plus dominantes en termes d'individus: les Irvingiaceae, les Combretaceae, les Rubiaceae et les Apocynaceae. Ce résultat peut s'expliquer par le fait que ces familles possèdent des semenciers attenants qui fructifient régulièrement le long des différentes routes secondaires. Il faut ajouter à cela, le rôle favorable de l'éclairage qui stimule la régénération naturelle de leurs diaspores (Yongo et al., 2013), accentué par le caractère héliophile des individus au stade juvénile de trois de ces familles Combretaceae (Terminalia superba), Rubiaceae (Nauclea diderrichii) et Apocynaceae (Alstonia boonei).

Le mode de dispersion dominant des diaspores en bordure de routes secondaires est l'anémochorie suivie de la zoochorie. Ce résultat est dû à l'ouverture du milieu qui fait place au potentiel floristique extérieur. Le vent et les animaux contribuent à près de $87 \%$ à la reconstitution des routes secondaires le long de ces 20 mètres par l'apport des diaspores extérieures provenant des semenciers attenant. Selon Missamba-Lola et al. (2015), la remarque a été faite que le retour à la forêt initiale d'une piste secondaire, après abandon, notamment en forêt à Marantaceae, ne semble être accéléré que par les animaux par dissémination des diaspores. 


\section{Conclusion}

La régénération naturelle des essences commerciales sur les routes secondaires de différents âges est effective dans l'UFA 09 003. Elle présente cependant une diversité en termes d'espèces plus prononcée sur les placettes vieilles de 15 ans d'âge et un nombre d'individus faibles sur l'ensemble de la zone d'étude. Pour favoriser la régénération naturelle des espèces sur les routes secondaires au moins un an après l'exploitation, les sociétés forestières devraient éviter l'envahissement des sites secondarisés par les graminées, favoriser la croissance des espèces héliophiles par des défrichements réguliers des bordures des routes secondaires, opérer un monitoring systématique des essences et sensibiliser les populations riveraines sur la nécessité de maintenir la dynamique de régénération de ces espèces.

\section{CONFLITS D'INTERETS} d'intérêts.

Les auteurs ne déclarent aucun conflit

\section{CONTRIBUTIONS DES AUTEURS}

EMAO a contribué à la conception et à la structuration de l'étude, à la collecte des données sur le terrain et à la rédaction de la première mouture de l'article. JBNM a contribué à la conception et à la structuration de l'étude. ACP a amendé et apporté les inputs du premier draft de l'article proposé par l'étudiant, a produit la deuxième version de l'article, l'intégration des corrections donnant lieu à la version finale. AMB a contribué aux corrections de la deuxième version et à la relecture de la version finale. DCM a contribué à la relecture de la version finale de l'article.

\section{REMERCIEMENTS}

Nous tenons particulièrement à remercier les encadreurs de terrain : Messieurs DJAMEN, MVOGO, OKEN, NGON, OYONO, KANGASSOUA, MANDJO, EVINA pour leur assistance technique, les doctorants du Département de Biologie et
Physiologie Végétales, en particulier CHIMI Cédric, ZEBAZE DONGMO Donatien, NJIKE Paméla pour leur contribution dans l'analyse des données et enfin les relecteurs qui ont participé à l'amélioration de la qualité de cet article.

\section{REFERENCES}

Akouehou GS, Lokossou RS, Lègba IS, Bio IC, Yaou LZ. 2017. Dynamique des peuplements des Parcelles d'Essai Sylvicoles (PES) de la forêt classée de la Sota au Nord-Est du Bénin. Int. J. Biol. Chem. Sci., 11(5) : 1994-2004. Doi : https://dx.doi.org/10.4314/ijbcs.v11i5.5.

Bele MY, Somorin O, Sonwa DJ, Nkem JN, Locatelli B. 2011. Politiques sur les Forêts et l'Adaptation aux Changements Climatiques au Cameroun. CIFOR, Bogor, Indonesie.

Doucet JL. 2003. L'alliance délicate de la gestion forestière et de la biodiversité dans les forêts du centre du gabon. Thèse de Doctorat, Faculté Universitaire des Sciences Agronomiques de Gembloux, p. 323 .

FAO. 2010. Prise en compte de la biodiversité dans les concessions forestières d'Afrique Centrale. Document de travail sur la biodiversité forestière $\mathrm{N}^{\circ} 1$. FAO, Rome, p.112.

Koubouana F, Ifo SA, Loupet LBM, Ndinga E. 2016. Diversité floristique et dynamique de reconstitution de la forêt du Parc Zoologique sous plantations à eucalyptus à Brazzaville, Congo. Int. J. Biol. Chem. Sci., 10(2): 609-619. Doi : http://dx.doi.org/10.4314/ijbcs.v10i2.13.

Marcon E. 2016. Mesure de la Biodiversité. UMR $\mathrm{E}_{\mathrm{CO}} \mathrm{F}_{\mathrm{O}} \mathrm{G}$, Guyane; 45-46.

MINFOF. 2016. Secteur forestier et faunique $\mathrm{du}$ Cameroun : faits et chiffres. MINFOF, 47.

Missamba-Lola AP, Matondo R, Marien JN, Samba-Kimbata MJ, Gillet JF. 2015. Dynamique spatio-temporelle des recrus 
forestiers aux bords des routes secondaires : Cas des UFA-CIB dans la cuvette Congolaise. Revue Scientifique et Technique Forêt et Environnement du Bassin du Congo, 4: 34-45.

Ndobe NS, Mantzel K. 2014. Déforestation, REDD et le Parc National de Takamanda au Cameroun-une étude de cas. Forest peuples programme charity, p. 48.

Ngueguim JR, Zapfack L, Noumi VN, Onana D, Betti JL, Riera B. 2015. Expériences sylvicoles au Cameroun : Croissance, mortalité et adaptabilité des espèces de bois d'œuvre dans la station forestière de Mangombé (1964-2010). Int. J. Biol. Chem. Sci., 9(6): 2789-2807. Doi : http://dxdoi.org/10.4314/ijbcs.v9i6.22.

Pandey SS, Cockfield G, Maraseni TN. 2016. Assessing the roles of community forestry in climate change mitigation and adaptation: A case study from Nepal.
Forest Ecology and Management, 360: 400-407. Doi.org/10.1016/j.foreco.2015. 09.040

Tchatchou B, Sonwa DJ, Ifo S, Tiani AM. 2015. Déforestation et Dégradation des Forêts dans le Bassin du Congo: État des Lieux, Causes Actuelles et Perspectives. Papier occasionnel 120. CIFOR, Bogor, Indonesie.

Tiebre MS, Ouattara D, Kpangui KB, Kouassi DF, N'guessan KE. 2016. Diversité floristique de la région de Foungbesso en zone de transition forêt-savane à l'ouest de la Côte d'Ivoire. Int. J. Biol. Chem. Sci., 10(3): 1007-1016. Doi : http://dxdoi.org/10.4314/ijbcs.v10i3.8.

Yongo OD, Kassi N'dja J, Tiebre MS, de Foucault B. 2013. Régénération naturelle des espèces ligneuses dans la foret de Ngotto (République Centrafricaine). Agronomie Africaine, 25(2): 105 - 11. 